\title{
Response to: "Zoledronate-induced acute anterior uveitis: a three-case report and brief review of literature"
}

\author{
Priya Samalia ${ }^{1}\left[\right.$ Rachael Niederer $^{1,2}$
}

Received: 28 July 2021 / Accepted: 3 August 2021 / Published online: 22 August 2021

(c) International Osteoporosis Foundation and National Osteoporosis Foundation 2021

\section{Dear Editor.}

We have read the article entitled "Zoledronate-induced acute anterior uveitis: a three-case report and brief review of literature" Jin et al., 2021. https://doi.org/10.1007/s11657021-00964-z with interest. We would like to thank the authors for this successful paper and make contributions based on our experience. Bisphosphonate induced ocular inflammation is topical and an important cause of uveitis.

In the current case series, Jin et al. described three cases of zoledronate-induced acute anterior uveitis. Acute anterior uveitis occurred $24-36 \mathrm{~h}$ after zoledronate infusion and all cases settled with topical steroid treatment. No cases were rechallenged with medication and none experienced recurrent inflammation during the study follow up period.

We recently reviewed cases of drug-induced ocular inflammation presenting to our uveitis service between 1st January 2008 and 1st January 2020 (Samalia P, Sims J, Niederer R. Drug-induced ocular inflammation. NZ Med J. 2020 Dec 18;133(1527):83-94. PMID: 33,332,330). The main class of drug responsible for drug-induced inflammation was bisphosphonates, occurring in 24 subjects (29 eyes), one of the largest series currently reported. This included 22 subjects ( 26 eyes) following zoledronate infusion and 2 subjects ( 3 eyes) on oral alendronate.

Of those with bisphosphonate-induced ocular inflammation in our series, 22 (91.7\%) subjects developed anterior uveitis and $2(8.3 \%)$ scleritis. Two $(9.1 \%)$ subjects were rechallenged with intravenous zoledronate and both developed recurrent uveitis on re-exposure. One subject with alendronate-induced uveitis developed recurrent uveitis 17.4 months after their initial episode when alendronate was not discontinued, with no further episodes on ceasing the drug.

Recurrence of uveitis following zoledronate rechallenge and with continued alendronate use provides evidence that stopping the inciting bisphosphonate is required for ocular inflammation to resolve.

Clinicians should be aware that bisphosphonates can induce uveitis. If bisphosphonate-induced uveitis goes unrecognized, the drug may be continued with chronic or recurrent inflammation and associated vision threatening uveitic complications.

\section{Declarations}

Conflict of interest The authors declare no competing interests.

Publisher's note Springer Nature remains neutral with regard to jurisdictional claims in published maps and institutional affiliations.

Priya Samalia

dr.priyasamalia@gmail.com

1 Ophthalmology Department, Auckland District Health

Board, Greenlane Clinical Centre, 214 Greenlane West, Epsom, Auckland 1051, New Zealand

2 Department of Ophthalmology, University of Auckland, 85 Park Road, Grafton, Auckland 1023, New Zealand 